\title{
Use of Transpulmonary Thermodilution to Measure Extravascular Lung Water Index in a Ventilated Patient
}

\author{
Gunjan L Shah, MD, Susan Ndicu, MD
}

A 61-year-old morbidly obese woman first presented to a community hospital with complaints of feeling "unwell" for 2 weeks. Her family reported that she had been having increasing fatigue, diarrhea, nausea, and vomiting for that period, as well as "dusky" fingers and toes. Prior to this time, she had noticed a runny nose, cough, and "bluish" toes for about 3 weeks. On presentation, she was hypotensive with a systolic blood pressure in the 50's mmHg and tachycardic to 115 beats per minute (bpm), but afebrile. Her creatinine was found to be $12 \mathrm{mg} /$ $\mathrm{dL}$ from an unknown baseline. Treatment was initiated in the Emergency Department with fluids and antibiotics, and she was admitted to the Medical Intensive Care Unit for management of septic shock. The patient was also found to have a urinary tract infection, with negative blood cultures. Stool was checked for Clostridium difficile infection, which was also negative. After 7 days of treatment for septic shock in the ICU including 2 days of hemodialysis, she was transferred to our tertiary center for further management.

The patient's past medical history included morbid obesity and obstructive sleep apnea, but no prior surgeries. Family history was remarkable for coronary artery disease in her father. She had no history of tobacco, ethanol or illicit substance use. She was not on any outpatient medications. Inpatient medications at the time of transfer included intravenous hydrocortisone, subcutaneous heparin, esomeprazole, regular insulin sliding scale and intravenous antibiotics. At the time of transfer, she was not requiring vasopressor medication support.

Physical examination showed an obese woman who was sedated and intubated, in no acute distress. She had a temperature of $95.9^{\circ} \mathrm{F}$, a heart rate of $108 \mathrm{bpm}$, a blood pressure of $118 / 55 \mathrm{mmHg}$, a respiratory rate of 24 breaths per minute, and an oxygen saturation of $94 \%$. She had a BMI of 42.3 . Notable physical findings were as follows: coarse breath sounds at bilateral lung bases, diminished bowel sounds, bilateral upper and lower extremity edema with cyanotic digits on all extremities and appearance of necrosis on several toes. She had a peripherally inserted central catheter (PICC) in the right upper extremity.

Studies on admission included the following: total white blood cell count of 16,600 with a left shift, hemoglobin of $9.9 \mathrm{~g} /$ $\mathrm{dl}$ and platelets 114,000. Chemistries were as follows: sodium $143 \mathrm{mmol} / \mathrm{L}$, potassium $3.7 \mathrm{mmol} / \mathrm{L}$, chloride $108 \mathrm{mmol} / \mathrm{L}$, bicarbonate $25 \mathrm{mmol} / \mathrm{L}$, blood urea nitrogen of $17 \mathrm{mg} / \mathrm{dl}$ and creatinine of $4.0 \mathrm{mg} / \mathrm{dl}$. A chest $\mathrm{x}$-ray showed multifocal consolidation with underlying bilateral pulmonary edema.

On the first day of admission the PICC line removed, and an internal jugular venous catheter and a femoral artery catheter were inserted. These were connected to a $\mathrm{PICCO}^{\circledR}$ (Pulsion Medical Systems, Munich, Germany) monitor for measurement of hemodynamic parameters as well as serial calculation of
Extravascular Lung Water Index (ELWI) via transpulmonary thermodilution technique. ELWI was from that point onward calculated serially, and used to direct the amount of intravenous fluid resuscitation and the use of vasopressors. With hemodialysis initiated, the ELWI was also used to determine the amount of fluid volume to be removed at each session. Clinical and X-ray resolution of the pulmonary edema were in turn monitored and correlated with the calculated ELWI. Ventilator settings were adjusted as needed to optimize oxygenation and ventilation depending on perceived extravascular lung water content. Other primary and surrogate hemodynamic parameters recorded included cardiac index $(\mathrm{CI})$, stroke volume index (SVI), stroke volume variation (SVV), mean arterial pressure (MAP), systemic vascular resistance index (SVRI), cardiac flow index (CFI), and global end diastolic volume index (GEDI).

\section{Discussion}

Fluid resuscitation in an ICU setting is a complicated affair because traditional cardiac monitoring does not always accurately predict fluid responsiveness. ${ }^{1}$ Specifically, a fluid challenge does not necessarily correlate with an increase in cardiac output (CO), and furthermore may not accurately reflect the development of pulmonary edema. For this reason, the measurement of extravascular lung water (EVLW) was developed. The PICCO system, developed by Pulsion Medical Systems from Munich, Germany, works by single thermal indicator transpulmonary dilution.

The main advantage of this system is that it requires only placement of a central venous and an arterial catheter, avoiding a pulmonary artery catheter and its associated risks. ${ }^{2}$ The central venous catheter and arterial catheter are connected to a pressure transducer and to the PICCO system. In difficult situations for catheter placement, such as in patients with a contraindication to the Trendelenburg position which makes placing a subclavian or internal jugular venous catheter tricky, the PICCO can still be used. This is unlike traditional Swan-Ganz catheters. One study has shown that the EVLW and CO parameters correlated even with a femoral venous catheter, demonstrating the efficacy of the PICCO to guide fluid status in patients that have central access issues. ${ }^{3}$

After calibration, ice-cold saline $\left(5^{\circ} \mathrm{C}\right)$ is injected in three $10 \mathrm{~mL}$ aliquots. The femoral arterial catheter measures the change in temperature through its thermistor tip. The subsequent pulse contour analysis calculates the area under the curve, the mean transit time (MTt), and the down-slope time (DSt). The mean transit time is the amount of time for half of the saline bolus to pass by the thermistor, while the down-slope time is the duration of the exponential decrease of the dilution curve. ${ }^{3}$

Using these measurements, the $\mathrm{CO}$, global end diastolic volume (GEDV), and intra-thoracic blood volume (ITBV) are calculated 


\begin{tabular}{|c|c|c|}
\hline Date & 2/21/09 & $2 / 21 / 09$ \\
\hline Time & 845 & 1645 \\
\hline \multicolumn{3}{|l|}{ CARDIAC OUTPUT } \\
\hline $\mathrm{Cl}(3-5 \mathrm{l} / \mathrm{min} / \mathrm{m} 2)$ & 2.37 & 2.52 \\
\hline \multicolumn{3}{|l|}{ Frequency } \\
\hline $\mathrm{HR}(1 / \mathrm{min})$ & 63 & 67 \\
\hline Rhythm & SR & SR \\
\hline \multicolumn{3}{|l|}{ Stroke Volume } \\
\hline SVI (40-60 ml/m2) & 34 & 37 \\
\hline \multicolumn{3}{|l|}{ Preload } \\
\hline GEDI $(680-800 \mathrm{ml} / \mathrm{m} 2)$ & 760 & 602 \\
\hline SVV (<10\%) & 3 & 10 \\
\hline \multicolumn{3}{|l|}{ Afterload } \\
\hline MAP $(\mathrm{mmHg})$ & 111 & 99 \\
\hline SVRI (1970-2390 dyn* $\left.{ }^{*}{ }^{*} \mathrm{~cm}-5^{\star} \mathrm{m} 2\right)$ & 3455 & 2986 \\
\hline \multicolumn{3}{|l|}{ Contractility } \\
\hline CFI (4.5-6.5 1/min) & 4.6 & 6 \\
\hline \multicolumn{3}{|l|}{ LUNG FUNCTION } \\
\hline ELWI (3-7 ml/kg) & 12 & 7 \\
\hline \multicolumn{3}{|l|}{ THERAPUTIC INTERVENTION } \\
\hline & \multicolumn{2}{|c|}{$3 \mathrm{~L}$ removed via $\mathrm{HD}$} \\
\hline
\end{tabular}

to estimate preload, and EVLW is calculated to indicate degree of pulmonary edema. ${ }^{2}$ These hemodynamic values are indexed to body surface area (BSA) by the DuBois formula ${ }^{2}$ with body weight in kilograms and height in centimeters squared. The area under the curve correlates with the CO. ${ }^{4}$ Multiplication of the $\mathrm{CO}$ and the DSt gives the pulmonary thermal volume (PTV), which is the largest individual volume in the series of indicator dilution mixing chambers. Intra-thoracic thermal volume (ITTV) is the volume of blood in which the ice-cold water is dissolved and is calculated by multiplying CO and MTt, which represents the total volume of the cardiac atria and ventricles and part of the systemic vascular blood volume or the right and left atrial end diastolic volumes (RAEDV and LAEDV), the right and left ventricular diastolic volumes (RVEDV and LVEDV), and the pulmonary thermal volume. ${ }^{2}$ Subtraction of PTV from ITTV represents the GEDV or the total diastolic blood volume of the heart. Previously, Sakka et al. determined the correlation of ITBV and GEDV to be $1.25 \mathrm{x}$ GEDV - 28.4(ml). ${ }^{5,6}$ EVLW can be calculated via this equation by subtracting ITTV from IBTV. ${ }^{4}$ Normal values of EVLW are $5-7 \mathrm{ml} / \mathrm{kg}$, with values above $10 \mathrm{ml} /$ $\mathrm{kg}$ correlating with mild pulmonary edema, and up to $30 \mathrm{ml} / \mathrm{kg}$ representing severe pulmonary edema. ${ }^{7}$

The principle of measuring EVLW rests in the fact that large increases in EVLW predate the onset of alveolar edema and symptoms of flooding. One study found that the transpulmonary thermodilution method is actually more accurate in detecting small rather than large changes in EVLW, which allows for earlier detection of pulmonary edema. This is explained by the fact that when large amounts of liquid are introduced, and the lung is already edematous, the ice cold water may not have spread throughout the appropriate volumes assumed in the above formulas allowing some introduced fluid to be undetected. ${ }^{4}$ Previous comments about the clinical use of extravascular lung water have questioned the use of PICCO given that the significance of detecting small increases in EVLW is unknown. ${ }^{6}$ We propose that utilization of PICCO in measuring EVLW in the patient described above allowed us to clinically decide, in conjunction with ventilatory status, arterial blood gases, and chest x-rays, the appropriate time to attempt extubation.

This patient had developed renal failure prior to arriving at our institution, and as such had negligible urine output while on dialysis allowing for near complete control of volume removal. We intervened on her fluid status directly by removing numerous liters of fluid per dialysis session. After inserting the PICCO system, our patient's EVLW was calculated to be $12 \mathrm{ml} /$ $\mathrm{kg}$, correlating with some pulmonary edema, and remained at this level until 2 liters were removed through hemodialysis. Immediately following this intervention, the EVLW decreased to $10 \mathrm{ml} / \mathrm{kg}$. Subsequent resuscitation efforts increased this value again to $12 \mathrm{ml} / \mathrm{kg}$. Our solution was to remove 3 liters of fluid on two subsequent days, decreasing the EVLW to $7 \mathrm{ml} / \mathrm{kg}$, or within normal range. The patient's chest xray and ventilatory status correlated with the improved values, and she was then successfully extubated.

\section{Conclusion}

Algorithmic decisions involving diagnosis and treatment of acute lung injuries are aided by the use of the PICCO system in measuring EVLW and may allow for the quantification of injury. Though several studies have not shown a correlation between chest $\mathrm{x}$-rays and EVLW values, ${ }^{6}$ we suggest that the volume changes within the lung may be enough to cause clinical difference even if the radiography is not significantly different, however formal prospective studies are necessary to analyze this relationship.

\section{References}

1. Perner, A and Faber, T. Stroke volume variation does not predict fluid responsiveness in patients with septic shock on pressure support ventilation. Acta Anaesthesiol Scand 2006; 50: 1068-1073

2. Mutoh, Tatsushi, et al. Goal-Directed Fluid Management by Bedside Transpulmonary Hemodynamic Monitoring After Subarachnoid Hemorrhage. Stroke. 2007;38:3218-3224

3. Schmidt, Sven, et al. Effect of the venous catheter site on transpulmonary thermodilution measurement variables. Crit Care Med 2007;35;3:783-786.

4. Fernandez-Mondejar, Enrique, et al. Small Increases in Extravascular Lung Water Are Accurately Detected by Transpulmonary Thermodilution. J Trauma. 2005;59:1420 - 1424

5. Sakka, SG, Rul CC, Pfeiffer UJ, et al. Assessment of cardiac preload and extravascular lung water by single transpulmonary thermodilution. Intensive Care Med. 2000; 26:180-187

6. Schuster, Daniel P. and Isakow, Warren. Extravascular lung water measurements and hemodynamic monitoring in the critically ill: bedside alternatives to the pulmonary artery catheter. Am J Physiol Lung Cell Mol Physiol 291:1118-1131, 2006

7. Bock, J and Lewis, F. Clinical relevance of lung water measurement. In: Practical applications of fiberoptics in critical care monitoring, edited by Lewis $\mathrm{F}$ and Pfeiffer U. Berlin: Springer-Verlag, 1990, p. $164-180$ 